\title{
Electric Field Simulations for Transcranial Brain Stimulation using FEM: an efficient implementation and error analysis
}

\author{
Saturnino, Guilherme Bicalho ; Madsen, Kristoffer Hougaard; Thielscher, Axel
}

Published in:

Journal of Neural Engineering

Link to article, DOI:

$10.1088 / 1741-2552 / \mathrm{ab} 41 \mathrm{ba}$

Publication date:

2019

Document Version

Peer reviewed version

Link back to DTU Orbit

Citation (APA):

Saturnino, G. B., Madsen, K. H., \& Thielscher, A. (2019). Electric Field Simulations for Transcranial Brain Stimulation using FEM: an efficient implementation and error analysis. Journal of Neural Engineering, 16(6), [066032]. https://doi.org/10.1088/1741-2552/ab41ba

\section{General rights}

Copyright and moral rights for the publications made accessible in the public portal are retained by the authors and/or other copyright owners and it is a condition of accessing publications that users recognise and abide by the legal requirements associated with these rights.

- Users may download and print one copy of any publication from the public portal for the purpose of private study or research.

- You may not further distribute the material or use it for any profit-making activity or commercial gain

- You may freely distribute the URL identifying the publication in the public portal 
ACCEPTED MANUSCRIPT

\section{Electric field simulations for transcranial brain stimulation using FEM: an efficient implementation and error analysis}

To cite this article before publication: Guilherme Bicalho Saturnino et al $2019 \mathrm{~J}$. Neural Eng. in press https://doi.org/10.1088/1741-2552/ab41ba

\section{Manuscript version: Accepted Manuscript}

Accepted Manuscript is "the version of the article accepted for publication including all changes made as a result of the peer review process, and which may also include the addition to the article by IOP Publishing of a header, an article ID, a cover sheet and/or an 'Accepted

Manuscript' watermark, but excluding any other editing, typesetting or other changes made by IOP Publishing and/or its licensors"

This Accepted Manuscript is @ 2019 IOP Publishing Ltd.

During the embargo period (the 12 month period from the publication of the Version of Record of this article), the Accepted Manuscript is fully protected by copyright and cannot be reused or reposted elsewhere.

As the Version of Record of this article is going to be / has been published on a subscription basis, this Accepted Manuscript is available for reuse under a CC BY-NC-ND 3.0 licence after the 12 month embargo period.

After the embargo period, everyone is permitted to use copy and redistribute this article for non-commercial purposes only, provided that they adhere to all the terms of the licence https://creativecommons.org/licences/by-nc-nd/3.0

Although reasonable endeavours have been taken to obtain all necessary permissions from third parties to include their copyrighted content within this article, their full citation and copyright line may not be present in this Accepted Manuscript version. Before using any content from this article, please refer to the Version of Record on IOPscience once published for full citation and copyright details, as permissions will likely be required. All third party content is fully copyright protected, unless specifically stated otherwise in the figure caption in the Version of Record.

View the article online for updates and enhancements. 


\title{
Electric Field Simulations for Transcranial Brain Stimulation using FEM: An Efficient Implementation and Error Analysis
}

\author{
Guilherme B Saturnino ${ }^{1,2}$, Kristoffer H Madsen ${ }^{1,3^{*}}$, and Axel Thielscher ${ }^{1,2^{*}}$
}

${ }^{1}$ Danish Research Centre for Magnetic Resonance, Centre for Functional and Diagnostic Imaging and Research, Copenhagen University Hospital Hvidovre, Hvidovre, Denmark.

${ }^{2}$ Department of Health Technology, Technical University of Denmark, Kgs. Lyngby, Denmark.

${ }^{3}$ Department of Applied Mathematics and Computer Science, Technical University of Denmark, Kgs.

Lyngby, Denmark.

* Shared last authorship

E-mail: axelt@drcmr.dk

\section{Abstract}

Objective: Transcranial magnetic stimulation (TMS) and transcranial electric stimulation (TES) modulate brain activity non-invasively by generating electric fields either by electromagnetic induction or by injecting currents via skin electrodes. Numerical simulations based on anatomically detailed head models of the TMS and TES electric fields can help us to understand and optimize the spatial stimulation pattern in the brain. However, most realistic simulations are still slow, and the role of anatomical fidelity on simulation accuracy has not been evaluated in detail so far.

Approach: We present and validate a new implementation of the Finite Element Method (FEM) for TMS and TES that is based on modern algorithms and libraries. We also evaluate the convergence of the simulations and estimate errors stemming from numerical and modelling aspects.

Main results: Comparisons with analytical solutions for spherical phantoms validate our new FEM implementation, which is three to six times faster than previous implementations. The convergence results suggest that accurately capturing the tissue geometry in addition to choosing a sufficiently accurate numerical method is of fundamental importance for accurate simulations.

Significance: The new implementation allows for a substantial increase in computational efficiency of FEM TMS and TES simulations. This is especially relevant for applications such as the systematic assessment of model uncertainty and the optimization of multi-electrode TES montages. The results of our systematic error analysis allow the user to select the best tradeoff between model resolution and simulation speed for a specific application. The new FEM code is openly available as a part of our open-source software SimNIBS 3.0.

Keywords: Transcranial Magnetic Stimulation, Transcranial Electrical Stimulation, Finite Element Method, Head Models, Volume conductor Models 


\section{Introduction}

Transcranial magnetic stimulation (TMS) and transcranial weak electric stimulation (TES) are the two best-established methods for non-invasive transcranial brain stimulation (TBS). Both use electric fields to modulate neural activity in a target brain region or a network of brain regions, but the two methods differ in the mechanism used to generate the field: TMS employs electromagnetic induction, while TES injects currents into the skin via surface electrodes. The ability to transcranially modulate brain activity without serious adverse effects makes TBS a valuable research tool in neuroscience $[1,2]$ and possibly also an effective treatment for several psychiatric and neurological diseases [3]. However, the physiological and behavioral TBS effects are still subject to large intersubject variability [4,5], which hampers the more wide-spread use of TBS in clinical applications [5].

As TMS and TES use electric fields as mechanism of action to modulate the membrane potential of the neural cells in the brain [3], inter-individual variations of the generated fields is likely a key factor that contributes to the observed physiological and behavioral variability. For both stimulation methods, the electric field distribution is strongly influenced by the anatomical distribution of the head tissues, often in complex and counter-intuitive ways [6-10]. Accurately modeling the electric field distribution generated in the brain, based on individualized models of the head anatomy, is thus important to establish a more stringent control of spatial targeting and dosing. In fact, it has been shown that computational models can help predict stimulationoutcome in the case of TMS [9].

There is an increasing interest in tools that perform electric field modelling for TBS [11-13]. However, field simulations with most of the popular packages such as our software SimNIBS (www.simnibs.org) still have high computational cost. A single simulation currently requires several minutes on a standard PC, which is inconvenient for the user. Equally important, this also slows down the development and broad adoption of more advanced applications of realistic and individualized field calculations that rely on the evaluation of many simulations, such as the optimization of multichannel TES montages [14-16] or the systematic uncertainty quantification of the simulation outcome [17-19]. There is also a lack of studies trying to quantify how much the error in modelling tissue boundaries impacts the accuracy in the electric field calculations, as previous studies addressing simulation accuracy have been focused on comparing different methods for TMS $[20,21]$ or TES [22] simulations rather than changes in the anatomical fidelity of the underlying head model.

In the current work, we present and validate a new implementation of the Finite Element Method (FEM) for TMS and TES that uses modern algorithms and libraries. We first present the basic mathematical equations that underlie the TMS and TES electric fields and discuss how they can be numerically solved using the finite element method (FEM). Afterwards, we describe and validate our new FEM implementation featured in the open source simulation software SimNIBS [11] version 3.0. We compare the new and the previous FEM implementations, demonstrating speed ups of three to six times and approximately two times lower memory requirements, without changes in accuracy or need for special hardware. We conclude with a demonstration of the relative contributions of numerical precision and anatomical fidelity of the modelled tissue boundaries to the overall simulation accuracy. 


\section{Methods}

\subsection{Equations for calculating the TMS and TES electric fields}

The equations governing the electric field $\boldsymbol{E}$ caused by a TMS coil are [23]:

$$
\begin{gathered}
\nabla \cdot(\underline{\sigma} \nabla \phi)=-\nabla \cdot\left(\underline{\sigma} \frac{\partial \boldsymbol{A}}{\partial t}\right) \\
\boldsymbol{E}=-\nabla \phi-\frac{\partial \boldsymbol{A}}{\partial t}
\end{gathered}
$$

The symbol $\underline{\sigma}$ denotes the tissue-specific ohmic conductivity, which can be either scalar or a $3 \times 3$ symmetric positive definite (SPD) tensor. In this study, we will assume scalar, piecewise-constant conductivities, with the values varying from $0.01 \mathrm{~S} / \mathrm{m}$ in bone to around $1.6 \mathrm{~S} / \mathrm{m}$ in cerebro-spinal fluid (CSF) [6]. $\boldsymbol{A}$ denotes the magnetic vector potential of the TMS coil, which depends on the coil's shape, position, and the current flow in the coil wires. The magnetic vector potential (more specifically, its temporal derivative) can be understood as the electric field which the TMS coil would induce in an infinite homogenous conductor. It is also sometimes referred to as the primary electric field. The symbol $\phi$ represents an electrical potential that can be understood as the source of a secondary electric field caused by tissue boundaries or, more generally, by variations in conductivity, partly counteracting the primary field. Additionally, we assume homogeneous Neumann boundary conditions in the entire boundary (that is, there is no current flow to the outside of the head). This model is based on the quasistatic approximation of Maxwell's equations at low frequencies, which can be safely applied for the TMS simulations $[24,25]$. This means that, while the current through the TMS coil varies over time, modifying the $A$ field and by consequence the electric field, we can separate $\boldsymbol{E}(\boldsymbol{r}, t)$ into two components such that $\boldsymbol{E}(\boldsymbol{r}, t)=\boldsymbol{E}(\boldsymbol{r}) \partial I(t) / \partial t^{.} \boldsymbol{E}(\boldsymbol{r})$ is a spatial component, calculated using Eq. 2, and $\partial I(t) / \partial t$ is a temporal component, given solely by the pulse shape.

In the case of TES, the electric potential is governed by a simple Laplace equation [26]:

$$
\begin{gathered}
\nabla \cdot(\underline{\sigma} \nabla \phi)=0 \\
\boldsymbol{E}=-\nabla \phi
\end{gathered}
$$

with $\phi$ being the electrical potential caused by the external stimulation, modelled by setting Dirichlet boundary conditions (that is, setting fixed electric potentials) on the electrode surfaces and homogeneous Neumann boundary conditions elsewhere. TES intensity is normally controlled by setting the current flow through the electrodes, and not by setting potential differences. To adjust the simulations to a given current flow, SimNIBS goes through the steps

1. Solve equation 3 setting the potential at an arbitrary reference electrode to $0 \mathrm{~V}$ and at an arbitrary active electrode surface to $1 \mathrm{~V}$.

2. With the solution, calculate the current flow through the cathode and through the anode.

3. Correct the solution such that the current flow matches the set value. This can be done by a simple linear scaling. 


\section{Repeat in the case 3 or more electrodes are present}

It is also possible to set-up the simulation using only Neumann boundary conditions, in which case the current calibration step would not be necessary. However, this assumes that the current flux through the entire electrode surface is constant, which might be a wrong assumption specially in large electrodes or when connecting many electrodes to a single stimulator channel. However, setting simulations with Neumann boundary conditions can be advantageous when dealing with many small and independently controlled electrodes or when building leadfield matrices for TES optimization [14].

When applying TES with alternating currents, the quasistatic approximation also applies [27]. This means that the electric field at any instant can be calculated by only taking into consideration the current flow in the electrodes at that instant. Additionally, the electric potential is linear with respect to the input currents. This means that we do not need to run a new simulation for each time point, and can instead scale and sum simulation results in the right proportions such that we obtain the desired currents through the electrodes and by consequence the resulting electric field.

Further information about the differential equations governing the TMS and TES electric fields can be found in the Supplementary Material A.

\subsection{Finite Element Method for Electric Field Simulations}

Equation 1 and 3, which give us the electric potentials for TMS and TES, respectively, have analytical solutions only in very simple geometries such as spheres [28]. To obtain the electric fields in a realistic head model, we must resort to numerical methods. Here, we apply the Finite Element Method (FEM) [29], a well-established numerical method for obtaining approximate solutions to partial differential equations.

We chose FEM because it offers an elegant and efficient framework to model complex geometries such as the human head. This is done first by discretizing the domain (such as the head) into small sections with simple geometric shape called elements. Here, we use tetrahedral elements, but usage of other shapes such as hexahedra is also possible. The elements do not overlap and share their vertices (or nodes) with several other elements. The discretized domain, defined by its nodes and elements is called a mesh. This simple approach is very effective in representing complicated geometries, such as the sulci and gyri in the human brain. FEM requires the choice of the type of functions (termed basis functions) that are used to model the spatial variations of the solution (i.e, the electric potential) within the domain. Here, we use nodal linear basis functions, i.e. we define one basis function per node per element. The basis function has a value of one in its corresponding node and decays linearly within each element, reaching a value of zero in the other element nodes. Outside the element, the basis function has a value of zero. This makes the problem computationally efficient as only immediate neighbors needs to be evaluated meaning that computations can be formulated with sparse matrices. Finally, we transform Equations 1 and 3 into their weak forms and use the Galerkin method to derive a system of equations based on the linear basis functions.

The steps above, described in detail in the Supplementary Material A, results in a system of equations of the form

$$
\boldsymbol{S u}=\boldsymbol{b}
$$


where $S$ is a sparse matrix of the size $N_{d o f} \times N_{d o f}$ and is also denoted as stiffness matrix. $N_{d o f}$ denotes the number of degrees of freedom, which roughly correspond to the number of nodes in the mesh in our case. $\boldsymbol{u}$ is the electric potential at the nodes and the right-hand side $\boldsymbol{b}$ contains information about boundary conditions and the $d \boldsymbol{A} / d t$ field in the TMS case.

By solving the TMS and TES systems, we obtain the electric potential. As the quantity of interest is the electric field $\boldsymbol{E}$, we need to compute the negative gradient of $\phi$ to obtain $\boldsymbol{E}$ in case of TES (eq. 4). For TMS, we have to sum the negative gradient of $\phi$ with the negative of the temporal derivative of the magnetic vector potential to obtain $\boldsymbol{E}$ (eq. 2). In our FEM implementation, that uses linear basis functions for the electric potentials, the results from the gradient calculations will be piecewiseconstant. That is, the electric field has a constant value within each element and is discontinuous across element boundaries. Because of these discontinuities, directly using the piecewise-constant gradients to approximate the generated electric fields will decrease the numerical accuracy of the simulations. To improve on the original solutions, we use the superconvergent patch recovery (SPR) procedure [30] to recover nodal values for the electric fields, and in obtain more accurate interpolations of the electric field values at any position in the head mesh. However, as the electric field is discontinuous across tissue boundaries due to the abrupt changes in conductivity, we calculate the recovered values for each tissue separately. The procedure is described in detail in the supplementary material A.

To solve the FEM system (Equation 5), SimNIBS 3.0 interfaces directly with PETSC [31], a powerful scientific computing library which offers a homogeneous interface to a large set of solvers and preconditioners with a low overhead. As the system of equations is symmetric positive definite in TES simulations or can be made positive definite in TMS simulations (see supplementary material A), we chose to use a preconditioned conjugate gradient (PCG) solver, with a relative error tolerance of $10^{-10}$ and the algebraic multigrid BommerAMG preconditioner from the hypre package [32], with HMIS coarsening, $V$ cycles, a maximum of 25 levels and 1 iteration per hypre call. This same type of preconditioner has previously been used in EEG and MEG forward modelling [33] and in EIT modelling including the complete electrode model [34].

\subsection{Spherical Phantom}

To validate our FEM implementation, we used a geometric model which consists of five concentric spherical shells of radii 75, 78, 80, 86 and $92 \mathrm{~mm}$ [20], shown in Figure 1. The shells emulate the outer boundaries of white matter (WM), gray matter (GM), cerebro-spinal fluid (CSF), skull and skin, respectively. Such a simple model allows us to calculate solutions analytically, and therefore directly evaluate numerical accuracy. We used six different resolutions for the sphere phantoms, shown in Table 1. The FEM solutions were compared to the analytical results by evaluating the electric fields at the nodes of a sphere surface with a radius of $76.5 \mathrm{~mm}$, which we term observation sphere in the following and which was embedded in the middle of the GM layer. The triangles of the observation sphere had a mean edge length of $0.64 \mathrm{~mm}$. FEM fields were evaluated by performing the FEM calculations and afterwards interpolating the electric fields on the observation sphere nodes using the SPR procedure.

We used the analytical solution developed by Sarvas [35] to validate our TMS FEM implementation. This solution gives the electric field produced by a magnetic dipole placed outside the sphere and is valid for any spherically symmetric conductivity distribution within the sphere. For the calculations, 
we positioned a magnetic dipole $15 \mathrm{~mm}$ above the surface of the sphere phantom and oriented it radially. In the FEM model, the conductivities of the two inner-most layers were set to $0.33 \mathrm{~S} / \mathrm{m}$, and the remaining conductivities were set to $1.79 \mathrm{~S} / \mathrm{m}, 0.01 \mathrm{~s} / \mathrm{m}$ and to $0.43 \mathrm{~S} / \mathrm{m}$ (stated from inside to the outside [36]). Please note that the detailed choice of the conductivities is not important for the purpose of model validation, as long as they fall roughly in the range relevant for the later applications.

For the TES FEM implementation, we used the formula developed by Rush and Driscoll [37] for calculating the electric fields caused by point electrodes in three concentric spherical shells. We used the same spherical models as for TMS but assigned to the three innermost shells a conductivity value of $0.33 \mathrm{~S} / \mathrm{m}$, making it into effectively a single shell. The model proposed by Rush and Driscoll features unrealistic point-wise electrodes. For this reason, instead of applying the electrodes directly to the surface of the spherical phantom, we choose to simulate the electric field in an "extended" spherical shell model with an outer radius of $102 \mathrm{~mm}$. We used the extended phantom to calculate the electric potentials at the outer surface of the original spherical phantoms, where we used the results as Dirichlet boundary conditions for the FEM calculations. Our aim was to mimic the boundary conditions caused by small (EEG-sized rather than standard TES-sized) electrodes as a worst-case test, as the numerical accuracy of ensuring the set boundary conditions is more difficult to guarantee for small surface areas with a low number of nodes. Visual inspection of the potentials in the model's outer layer confirmed that this procedure corresponds approximately to simulating a circular electrode of $12 \mathrm{~mm}$ diameter.

Using the results obtained with the analytical models as reference, we assessed the error of the FEM solution in the observation sphere as

$$
\epsilon=\sqrt{\frac{\int\left\|\boldsymbol{E}_{\mathrm{ref}}-\boldsymbol{E}_{\mathrm{fem}}\right\|^{2} d \Omega}{\int\left\|\boldsymbol{E}_{\mathrm{ref}}\right\|^{2} d \Omega}} \times 100 \%
$$

where $\boldsymbol{E}_{\text {ref }}$ is the reference electric field on the observation sphere, and $\boldsymbol{E}_{\text {fem }}$ the electric field obtained from the FEM calculations in SimNIBS. 


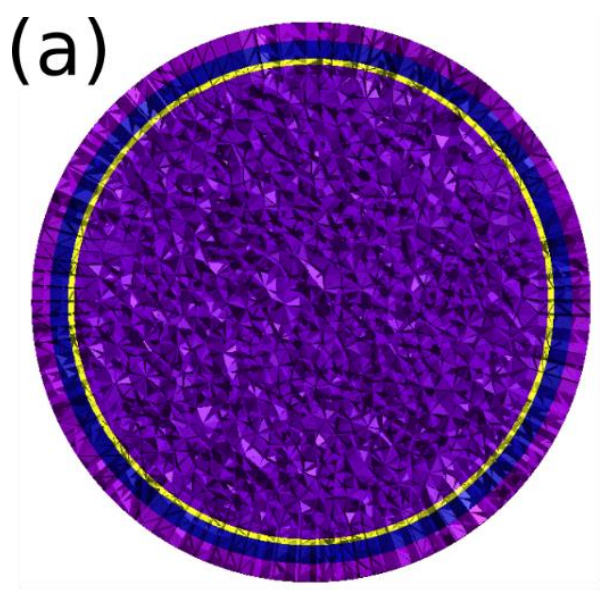

\section{(b)}

Conductivity $(\mathrm{S} / \mathrm{m})$

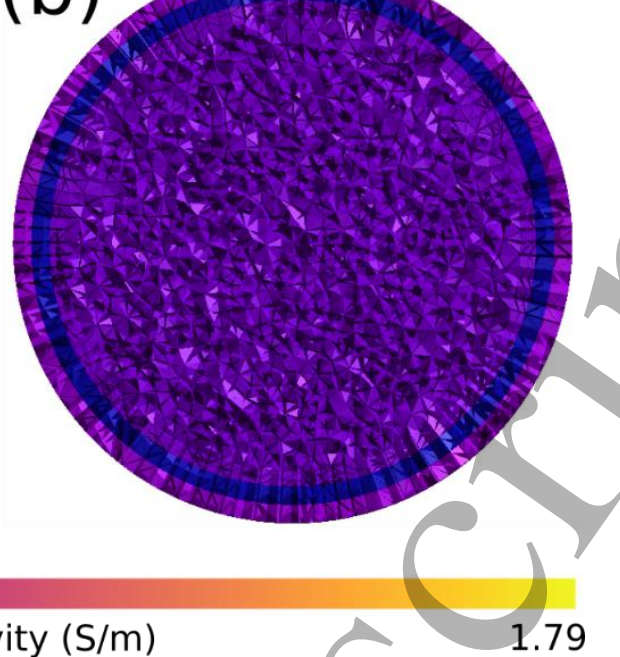

1.79

Figure 1: Conductivity distributions of the spherical phantoms for testing (a) TMS and (b) TES simulations.

Table 1: Number of Nodes, density of nodes in the spherical surfaces, and mean edge length for the tetrahedral elements in the spherical phantoms.

\begin{tabular}{cccc}
\hline $\begin{array}{c}\text { Number of } \\
\text { nodes }\left(\times 10^{6}\right)\end{array}$ & $\begin{array}{c}\text { Number of } \\
\text { tetrahedra }\left(\times 10^{6}\right)\end{array}$ & $\begin{array}{c}\text { Mesh density, } \\
\text { nodes } / \mathbf{m m}^{\mathbf{2}}\end{array}$ & $\begin{array}{c}\text { Mean edge } \\
\text { length, } \mathbf{~ m m}\end{array}$ \\
\hline $\mathbf{0 . 0 5}$ & 0.26 & 0.07 & 5.1 \\
\hline $\mathbf{0 . 1 2}$ & 0.72 & 0.14 & 3.6 \\
\hline $\mathbf{0 . 3 0}$ & 1.80 & 0.28 & 2.7 \\
\hline $\mathbf{0 . 8 4}$ & 5.05 & 0.55 & 1.9 \\
\hline $\mathbf{2 . 7 0}$ & 16.43 & 1.21 & 1.3 \\
\hline $\mathbf{7 . 2 7}$ & 44.63 & 2.41 & 0.92 \\
\hline
\end{tabular}

\subsection{Realistic Head Model}

To evaluate the performance of our FEM implementation as well as estimate the error and its causes, we ran simulations on realistic head models created in SimNIBS 2.1 using the headreco pipeline [38], based on magnetic resonance (MR) T1 and T2-weighted anatomical images of a single subject (see [6] for details regarding the image acquisition parameters). It has six tissue types corresponding to WM, GM, CSF, skull, skin and eyes (Figure 2(a)), and accounts for the major air cavities by sparing them in the mesh (effectively treating them as non-conducting vacuum). Tissue conductivities were set to 0.126 (WM), 0.275 (GM), 1.654 (CSF), 0.01 (skull), 0.465 (scalp) and 0.5 (skin) $\mathrm{S} / \mathrm{m}$, respectively [6].

In the TMS simulation, we calculated the electric field induced by a MagStim 70mm coil over the motor cortex, as shown in Figure 2(b). The current flux ( $d I / d t)$ was set to $1 \mathrm{~A} / \mu \mathrm{s}$. In the TES simulations, we calculated the electric field caused by a $50 \times 50 \mathrm{~mm}$ anode placed over the motor cortex and a 70x50mm supraorbital cathode, as shown in Figure 2(c). The current flux was set to 1 $\mathrm{mA}$, and the electrode conductivity was set to $1 \mathrm{~S} / \mathrm{m}$. The potential in the electrodes was set homogeneously in the entire electrode surface, which corresponds to having a highly conductive material (such as a metal mesh) on the upper layer of the electrode. 
During the meshing procedure, individual surfaces representing each tissue are extracted and, using meshfix [39], cleaned from self-intersections, decoupled from each other, re-meshed at a given density to ensure good triangle quality and slightly smoothed using the Taubin algorithm [40] to reduce artifacts caused by voxelization. Using the re-meshing step, we can control the anatomical accuracy of the head model by changing the target node density for the resampling of the reconstructed surfaces (measured as nodes $/ \mathrm{mm}^{2}$ ). This way, we obtain an insight into the node density required to guarantee a sufficiently accurate representation of the anatomical structures in the head and, by consequence, of the simulation results. Figure 3 shows in the left column a part of the GM surface around the left motor cortex and on the right column a cut of the CSF, GM and WM volumes in the same region, for each head model, and Table 2 shows the mesh properties of each model. We see that, as node density increases, the thin sulci become deeper and finer geometric details are resolved. Please notice that, while the mesh stems from an MRI image of $1 \mathrm{~mm}^{3}$ resolution, the CAT12 cortex segmentation can capture sub-millimeter features by leveraging the continuity of the cortex boundaries across adjacent voxels [41] and the meshing procedure in SimNIBS involves an appropriate level of surface smoothing [26]. Therefore, we do not observe a voxel-like structure in the mesh, even at the finest resolution.

Increasing node density leads to not only to increased anatomical accuracy, but also to smaller tetrahedral elements. Both effects contribute to increase the accuracy of the simulated fields. To decouple both of these effects, we have in addition refined the four least dense head meshes by splitting their constituent tetrahedral elements. The meshes of density $0.125,0.25$ and 0.5 nodes $/ \mathrm{mm}^{2}$ were refined twice, while the mesh of density 1.0 node $/ \mathrm{mm}^{2}$ was refined once due to computer hardware constraints. These head meshes have the same anatomical accuracy in representing the tissues, but the much finer elements allow for more accurate FE basis functions. The properties of these meshes are listed in Table 3. As each refinement decreases the size of the elements by about half and the numerical accuracy of a first order method is approximately linearly related to the element size [29], we expect the true numerical error $\epsilon^{*}$ to be $\epsilon \leq \epsilon^{*} \leq 5 / 4 \epsilon$ in the first three meshes and $\epsilon \leq \epsilon^{*} \leq 3 / 2 \epsilon$ for the mesh, where $\epsilon$ is the error estimated with the refined mesh using Eq. 6.

We used the modified Hausdorff distances [38,42] to quantify how well the gray matter surfaces that had been resampled at different node densities matched to the surface with the reference node density $\left(2.0\right.$ nodes $\left./ \mathrm{mm}^{2}\right)$. As the modified Hausdorff distance is dependent on the surface density, which diminishes as density increases, we used the refined versions of the gray matter surfaces described above to evaluate the modified Hausdorff distances. As the meshes were refined by splitting, the surface shape identical to the original shape. We obtained values of $0.57,0.33,0.30$ and $0.29 \mathrm{~mm}$ for densities of $0.125,0.25,0.5$ and 1.0 nodes $/ \mathrm{mm}^{2}$, respectively.

As MR quality can affect the precision of the segmentations [38], we additionally tested the effect of lowering MR image resolution from $1.0 \mathrm{~mm}^{3}$ to $1.5 \mathrm{~mm}^{3}$ and $2.0 \mathrm{~mm}^{3}$ in the TMS and TES simulation error. The modified Hausdorff distances for the gray matter surfaces obtained from the downsampled images were 0.46 and $0.48 \mathrm{~mm}$, and thus in a similar range as those stated above for the lower-resolution meshes. The details of this test are covered in Session B1 of the Supplementary Material B. 
Additionally, we compared the new FEM implementation with SimNIBS 2.1, which is a popular software package for calculating TES and TMS electric fields [11] and which uses GetDP [43] to form and solve the FEM system. GetDP is a general environment for FEM problems, offering great flexibility in setting up and solving a large range of PDEs. In SimNIBS 2.1, GetDP is configured to use the CG method with a relative error of $10^{-10}$ and the incomplete cholesky preconditioner with two factor levels. Our aim was to confirm that the new implementation produces the same results as SimNIBS 2.1, and to quantify the improvements in computational efficiency and memory consumption.

(a)

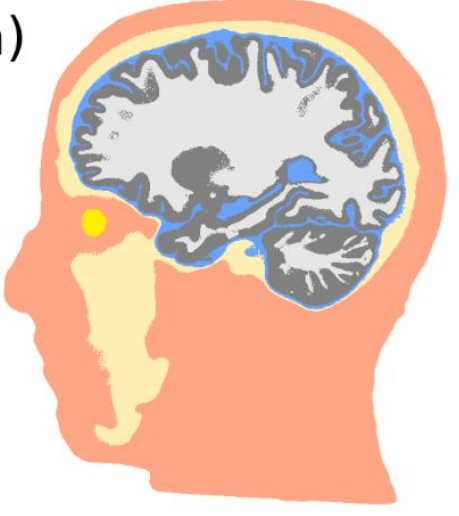

(b)

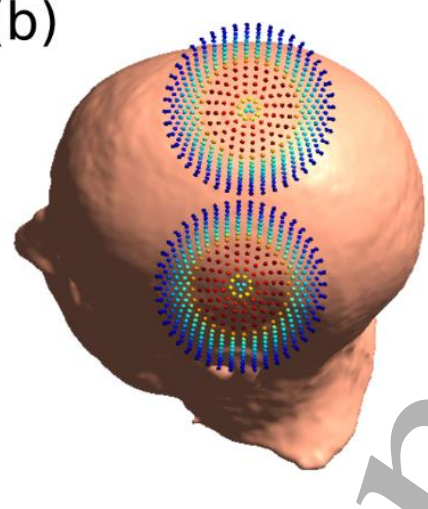

(c)

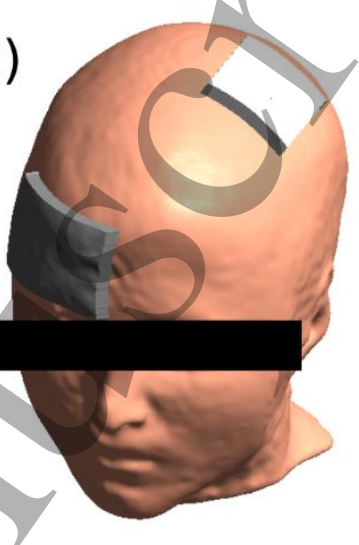

Figure 2: (a) Head Model with six tissues: white matter (WM, white), grey matter (GM, gray), cerebrospinal fluid (CSF, blue), skull (beige), skin (pink) and eyes (yellow). (b) Coil in TMS simulations. (c) Electrode montage for TES simulations. The mesh with a node density of 0.5 nodes $/ \mathrm{mm}^{2}$ is shown as an example. 


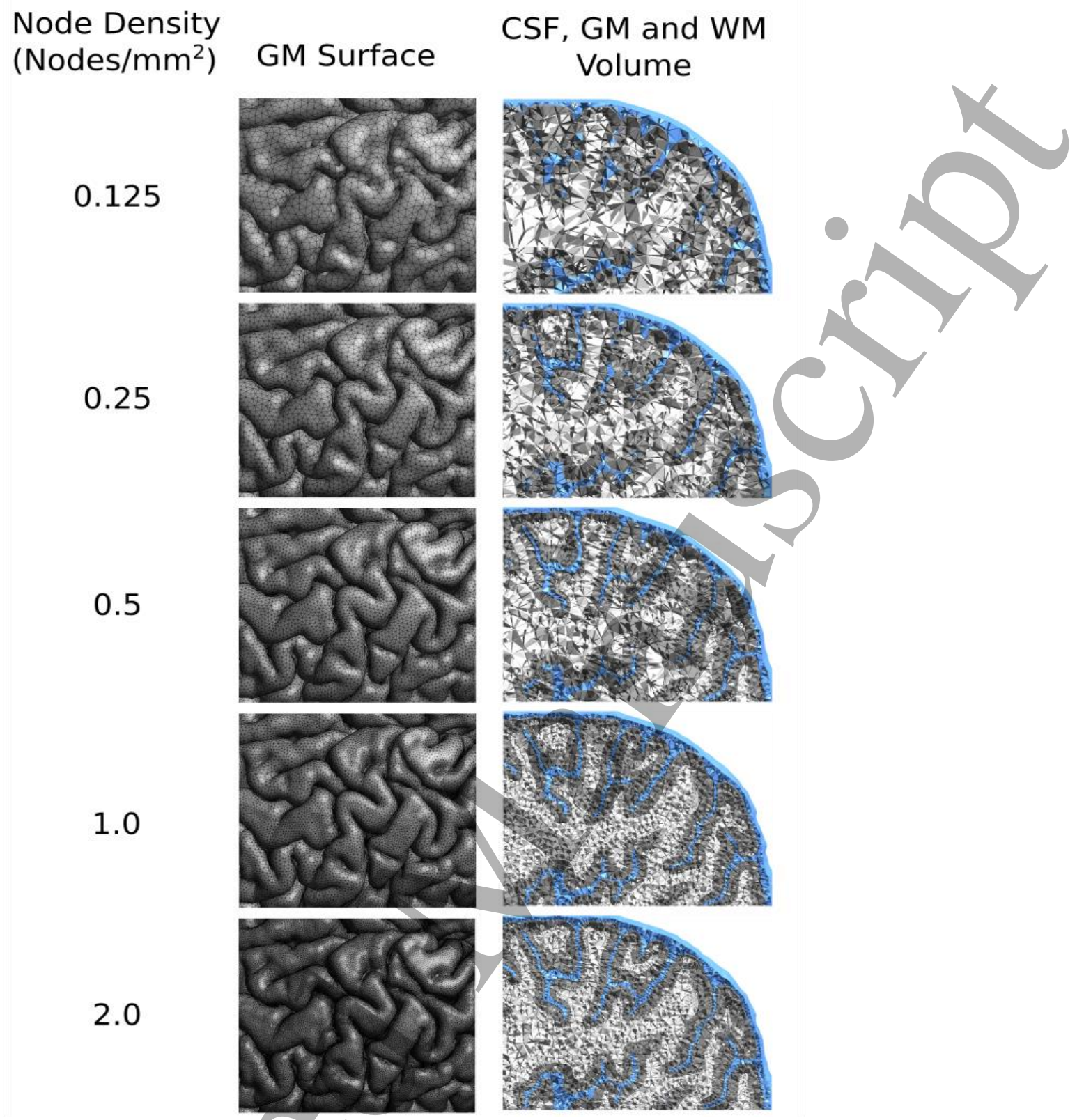

Figure 3: GM surface and GM, WM and CSF volumes at increasing mesh resolutions. Notice that not only the elements become finer, but also that the anatomical details of the CSF/GM boundary in the sulci are better preserved at higher mesh resolutions. 
Table 2: Nominal and achieved node densities in the surfaces, number of nodes, number of tetrahedra and mean edge length in the tetrahedral elements for the five head models of different resolutions.

\begin{tabular}{lllll}
\hline $\begin{array}{l}\text { Nominal } \\
\text { Density } \\
\left(\text { Nodes } / \mathrm{mm}^{2}\right)\end{array}$ & $\begin{array}{l}\text { Achieved } \\
\text { Density } \\
\left(\text { Nodes } / \mathrm{mm}^{2}\right)\end{array}$ & $\begin{array}{l}\text { Number of } \\
\text { nodes }\left(\times 10^{6}\right)\end{array}$ & $\begin{array}{l}\text { Number of } \\
\text { tetrahedra } \\
\left(\times 10^{6}\right)\end{array}$ & $\begin{array}{l}\text { Mean edge } \\
\text { length }(\mathrm{mm})\end{array}$ \\
\hline
\end{tabular}

\begin{tabular}{ccccc}
\hline $\mathbf{0 . 1 2 5}$ & 0.149 & 0.13 & 0.7 & 3.63 \\
\hline $\mathbf{0 . 2 5}$ & 0.275 & 0.30 & 1.7 & 2.28 \\
\hline $\mathbf{0 . 5}$ & 0.520 & 0.68 & 3.5 & 2.15 \\
\hline $\mathbf{1 . 0}$ & 0.996 & 2.0 & 11.2 & 1.48 \\
\hline $\mathbf{2 . 0}$ & 2.001 & 3.3 & 18.5 & 1.23 \\
\hline
\end{tabular}

Table 3: Mesh density data for the refined versions of the head meshes. Please notice that the first three meshes were refined twice, while the last mesh was only refined once.

\begin{tabular}{ccccc}
\hline $\begin{array}{l}\text { Original Density } \\
\left(\text { Nodes } / \mathbf{m m}^{2}\right)\end{array}$ & $\begin{array}{l}\text { Refined mesh } \\
\text { Density } \\
\left(\text { Nodes } / \mathbf{m m}^{2}\right)\end{array}$ & $\begin{array}{l}\text { Number of } \\
\text { nodes in } \\
\text { refined } \\
\text { mesh }\left(\times \mathbf{1 0}^{6}\right)\end{array}$ & $\begin{array}{l}\text { Number of } \\
\text { tetrahedra in } \\
\text { refined mesh } \\
\left(\times 10^{6}\right)\end{array}$ & $\begin{array}{l}\text { Mean edge } \\
\text { length in } \\
\text { refined } \\
\text { mesh }(\mathbf{m m})\end{array}$ \\
\hline $\mathbf{0 . 1 2 5}$ & 2.376 & 8.0 & 47.2 & 0.98 \\
\hline $\mathbf{0 . 2 5}$ & 4.398 & 18.5 & 109.5 & 0.76 \\
\hline $\mathbf{0 . 5}$ & 8.321 & 38.3 & 225.9 & 0.58 \\
\hline $\mathbf{1 . 0}$ & 4.036 & 15.2 & 89.4 & 0.78 \\
\hline
\end{tabular}

\subsection{Sulcus Phantom}

We calculated the electric field in a simplified representation of a sulcus (Figure 4a) in order to explore the influence of element size and anatomical features on the numerical accuracy in more detail. As this model has an analytical geometric description, this approach allowed us to keep a good anatomical fidelity of the surfaces while changing element size, as well as changing the model geometry while keeping the element size. The model was composed of bone, CSF, GM, and WM. The model dimensions were set to mimic those of a real sulcus, and then sufficiently extended along the $X$ and $Z$ dimensions to ensure that the vertical boundaries did not have a dominant influence on the field distribution in the center of the phantom. The sulcus was parametrized using a line and a semicircle on each side. Conductivities were set to the same values as used the head models.

We simulated two positions for the TMS coil, with the coil being positioned $40 \mathrm{~mm}$ above the bone layer and oriented perpendicular (Figure 4b) or parallel (Figure 4c) to the sulcus. We also evaluated two cases in which we aimed to roughly mimic the field of an unfocal TES set-up. For that, we set potentials along the upper and lower boundaries of the model (Figure 4d) or along its left and right vertical boundaries (Figure 4e). For each case, we simulated the field in six models with increasing resolution (Table 4).

To quantify how misrepresentations of the sulcus affect the overall error, we created four additional models with a high mesh density, but with an altered sulcus geometry. These models are described in detail in Session B2 of the Supplementary Material B. 
We visualized the field and numerical errors in a region of interest of the gray matter contained in an $80 \mathrm{~mm} \times 20 \mathrm{~mm}$ box along the $X$ and $Z$ directions, placed in the center of the model. For each simulation, we interpolated the electric fields at the positions corresponding to the barycenters of the tetrahedra of the highest resolution model using the SPR procedure. Similarly as for the head models, we calculated the errors as the norm of the difference between the interpolated electric fields and the fields of the reference model.

(a)

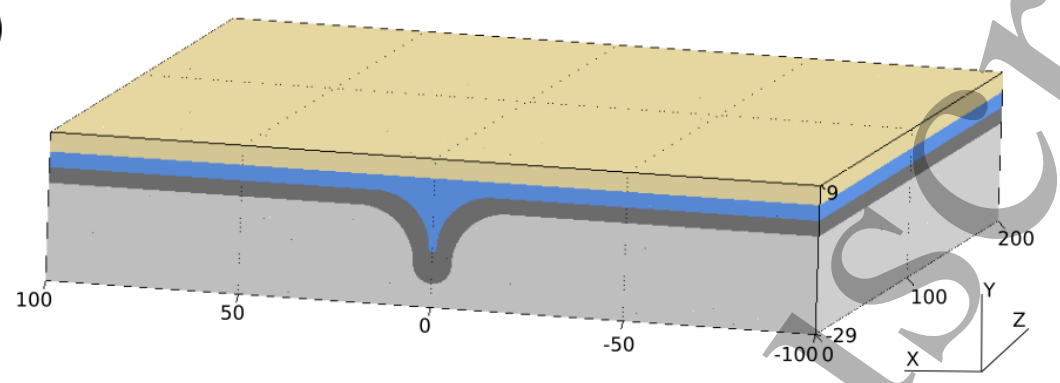

(b)

(c)
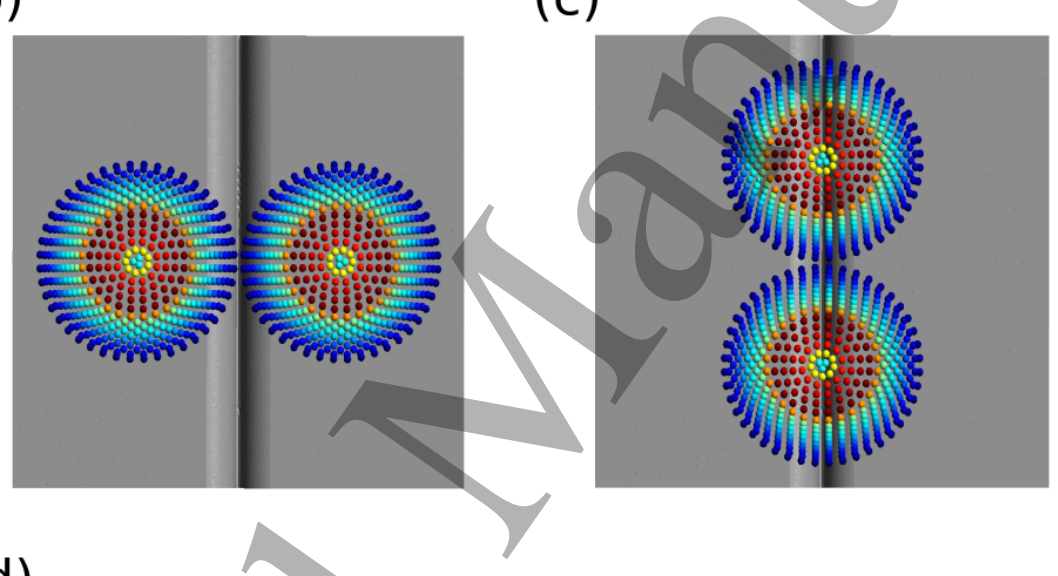

(d)

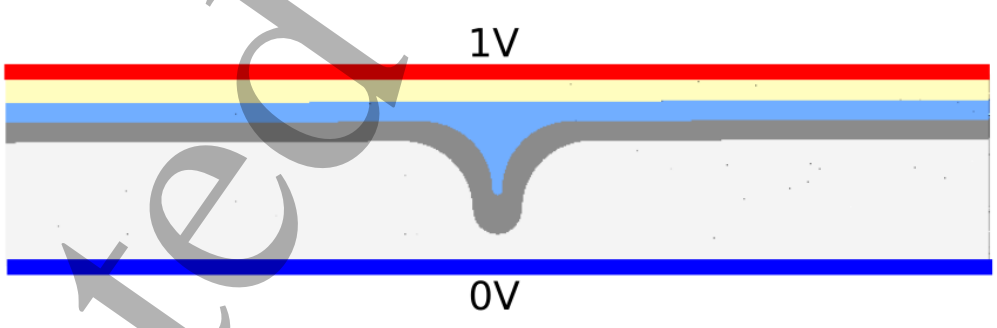

(e)

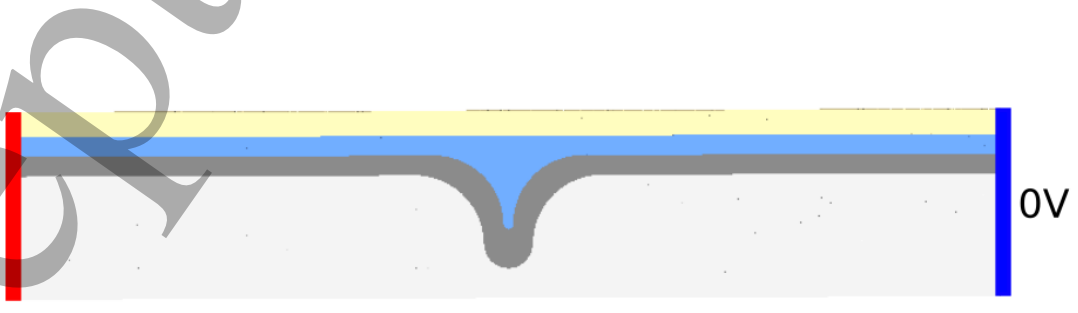

Figure 4: (a) Gyrus phantom with skull (beige), CSF (blue), GM (grey) and WM (white).

Dimensions are shown in $\mathrm{mm}$. (b) TMS simulation with the strongest fields induced parallel to the sulcus. (c) TMS simulation with the strongest fields induced perpendicular to the sulcus. (d) TES simulation with the potentials set at the horizontal boundaries at the top and bottom of the model. (e) TES simulation with the potentials set at two of the horizontal sides. 


\section{Results}

Table 4: Number of Nodes, density of nodes in the gray matter surface and mean edge length of the tetrahedral elements for the sulcus models.

\begin{tabular}{cccc}
\hline $\begin{array}{c}\text { Number of } \\
\text { nodes }(\times \mathbf{1 0})\end{array}$ & $\begin{array}{c}\text { Number of } \\
\text { tetrahedra } \\
\left(\times \mathbf{1 0}^{\mathbf{6}}\right)\end{array}$ & $\begin{array}{c}\text { Mesh density } \\
\left(\text { nodes } / \mathbf{m m}^{2}\right)\end{array}$ & $\begin{array}{c}\text { Mean edge } \\
\text { length }(\mathbf{m m})\end{array}$ \\
\hline $\mathbf{0 . 0 5}$ & 0.26 & 0.13 & 3.8 \\
\hline $\mathbf{0 . 0 8}$ & 0.43 & 0.18 & 3.2 \\
\hline $\mathbf{0 . 1 5}$ & 0.77 & 0.30 & 2.7 \\
\hline $\mathbf{0 . 3 0}$ & 1.6 & 0.53 & 2.0 \\
\hline $\mathbf{0 . 8 2}$ & 4.4 & 1.16 & 1.4 \\
\hline $\mathbf{1 . 7 2}$ & 9.4 & 2.1 & 1.1 \\
\hline & & & \\
& & & \\
\end{tabular}

\subsection{Spherical Phantom}

Figure 5 shows the error obtained in the TMS and TES electric fields for the six sphere models with increasing mesh density, with and without the SPR recovery step. We see that the errors monotonically decay and in the finest model investigated are below $1 \%$ in TES and below $0.1 \%$ (with SPR) in TMS, validating the FEM implementation. We also see that TES electric field has a larger error than the TMS field, probably because the TES electric field is solely determined by the gradient of the potential, calculated using FEM, while the TMS electric field is also strongly dependent on the magnetic vector potential field which is calculated with a higher accuracy due to its simple analytical form. Also, we see that SPR recovery reduces the error in all cases, but the reduction is markedly stronger in TMS, likely because the electric field produced by TMS decays at a faster rate than the TES electric field.

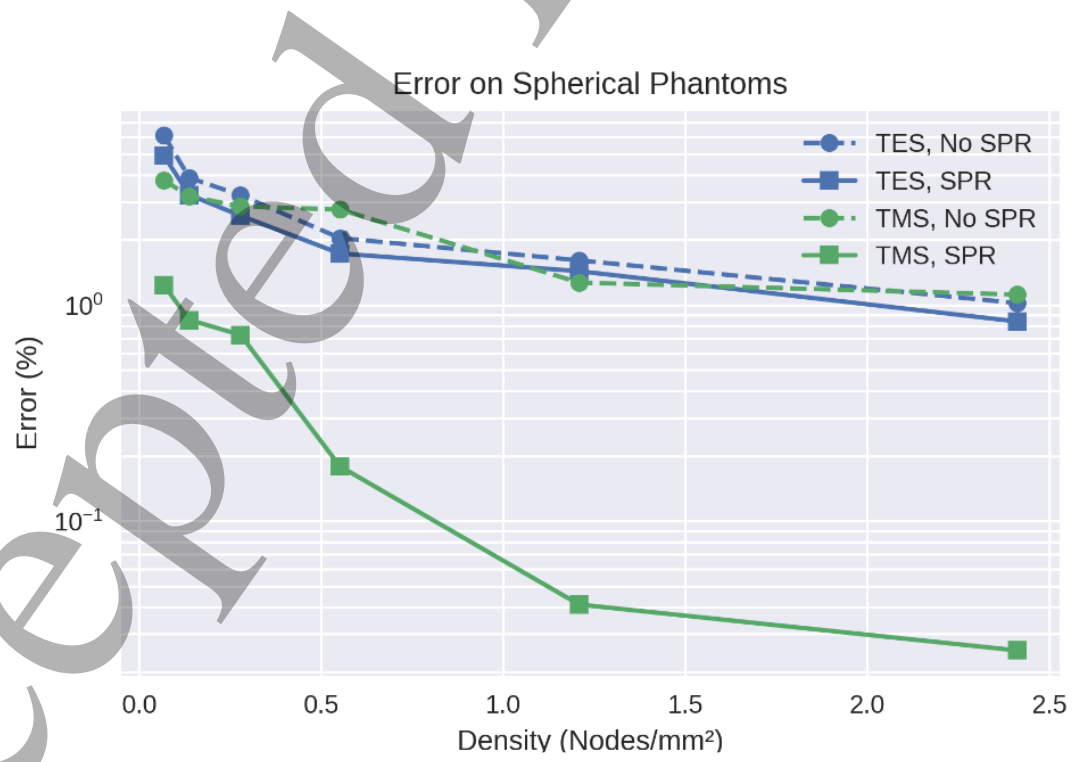

Figure 5: Error in the TMS and TES electric fields simulations for spherical phantoms, with and without the SPR recovery step. The FEM results converge with increasing mesh density and show good agreement with the analytical solutions. 


\subsection{Realistic Head Model}

\subsubsection{Error Analysis}

Figure 6 shows the electric field on this middle cortical surface for the different mesh resolutions, interpolated using the SPR procedure. It also shows the errors, defined as the norm of the difference between the electric field obtained at each of the four lower resolutions and the field obtained at the highest resolution, at each position.

Even the results obtained for the lowest mesh density of 0.125 nodes $/ \mathrm{mm}^{2}$ give a good overview of the overall field distribution in $\mathrm{GM}$, suggesting that this density might be adequate for qualitative visualizations. However, the absolute errors in the estimated field strengths are still high (see the following paragraphs for details). At 0.5 nodes $/ \mathrm{mm}^{2}$, the values for the maxima of the electric fields seems to be better resolved, indicating this model is better suited for quantitative analysis.

We used Equation 6 to quantify the errors with the electric field obtained with the highest resolution model as $\boldsymbol{E}_{\text {ref. }}$. To understand whether the improvement in simulation accuracy with increasing mesh resolution was primarily driven by the reduced size of the tetrahedral elements and therefore improved numerical accuracy, or by the better anatomical fidelity in representing the GM/CSF and other tissue boundaries, we also evaluated the error on the models refined by splitting (described in Section 2.5). In addition, in to evaluate the effect of the interpolation method, we used both the SPR-based interpolation and the unprocessed, element-wise constant electric fields. This comprehensive analysis, shown in Figure 7, allowed us to identify the main factors underlying simulation accuracy.

Generally, errors were high at the lowest mesh density, clearly exceeding $10 \%$ for all cases. Employing the SPR-based interpolation substantially improved the field estimates and helped to achieve errors around $6 \%$ for TMS and $8 \%$ for TES for a mesh density of 0.5 nodes $/ \mathrm{mm}^{2}$. This is the standard mesh density in the headreco pipeline and was selected as tradeoff between acceptable simulation time and numerical error. Refining the mesh also improves the solution by a similar extent as by applying SPR, as both have the same effect of increasing numerical accuracy. By applying SPR to the refined mesh, we only obtain small gains in accuracy. The exception is the mesh with a density of 1.0 node $/ \mathrm{mm}^{2}$, which was refined only once (due to memory limitations) while the other meshes were refined twice. Applying SPR to this refined mesh improves the solution to the level obtained by applying SPR to the unrefined mesh. Taken together, this indicates that increasing the numerical accuracy of the simulations does improve the solution. However, the error quickly reaches a lower bound, which is set by the anatomical fidelity of the model, rather than by the employed numerical method. This shows that improvements in numerical accuracy do not necessarily translate into a better overall accuracy.

The errors caused by down-sampling the input image from $1 \mathrm{~mm}^{3}$ to $1.5 \mathrm{~mm}^{3}$ and $2.0 \mathrm{~mm}^{3}$, shown in Table B2 of the Supplementary Material B, are in the same range ( $9 \%$ to $13 \%$ in the studied cases) as observed here for the lower density models tested. This indicates that, in addition to having good quality surface reconstructions, it is also fundamental to have high-quality input images so that the gray matter geometry can be accurately captured by the segmentation pipeline.

As it is still of interest to know the numerical accuracy of our simulations, we compared the fields obtained with the original and refined versions of the same head meshes, interpolated in the middle 
of the gray matter cortex. The results, shown in Fig. 8, reveal a clear improvement in numerical accuracy when employing finer meshes, with larger gains when the density is low. At a density of 1.0 node $/ \mathrm{mm}^{2}$, we obtain errors of $2.6 \%$ for TMS and $2.9 \%$ for TES, close to the $2 \%$ threshold proposed by [21].

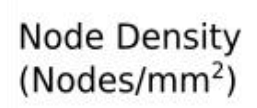

\section{TMS}

0.125
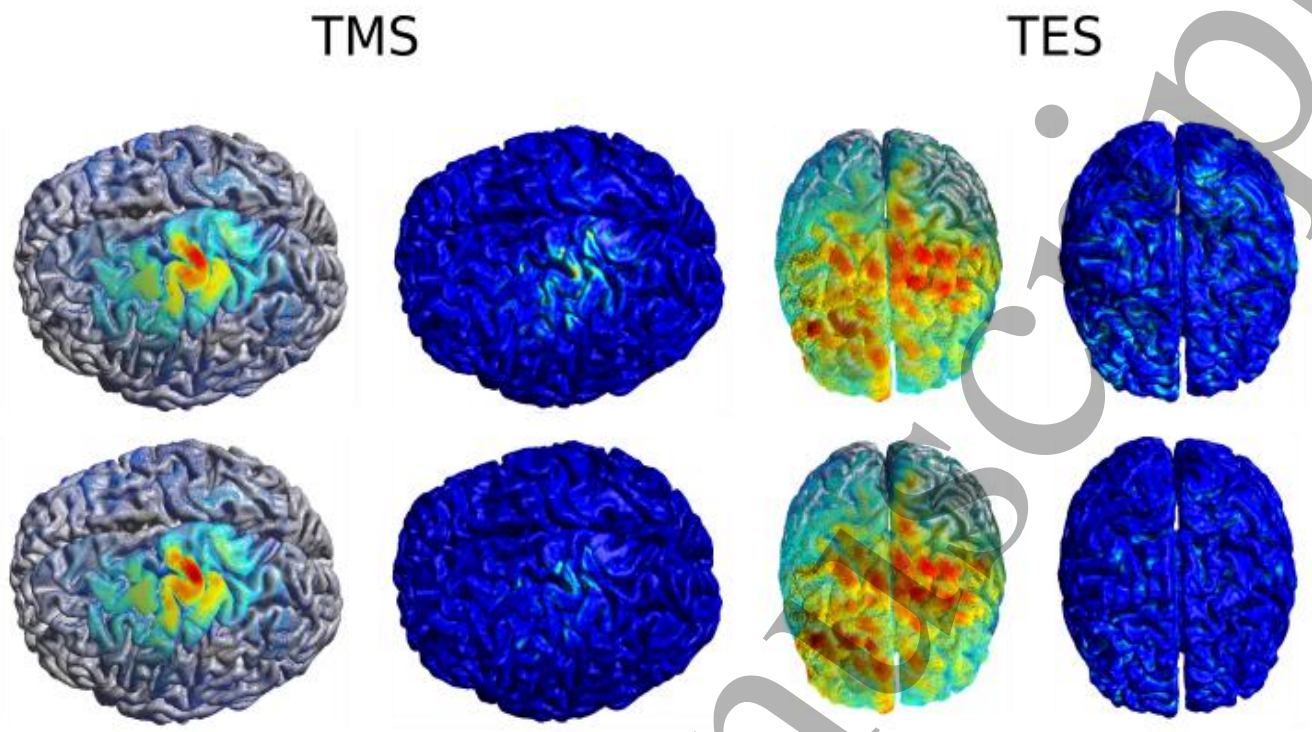

0.5
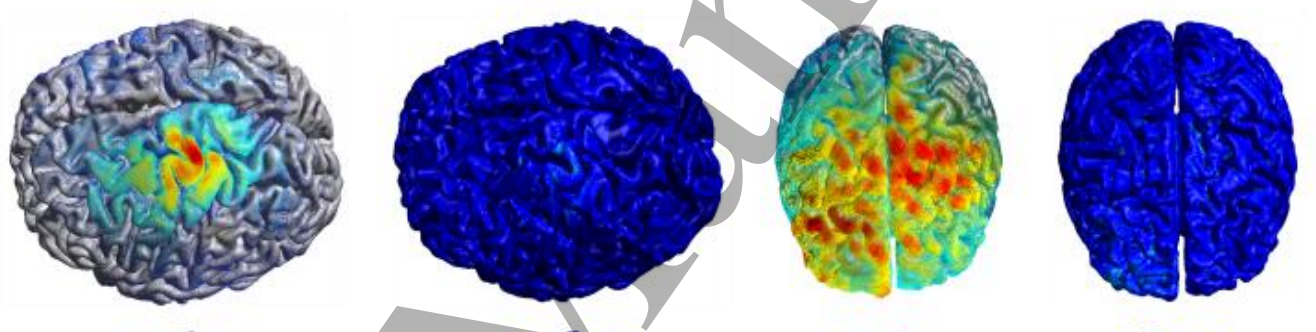

1.0
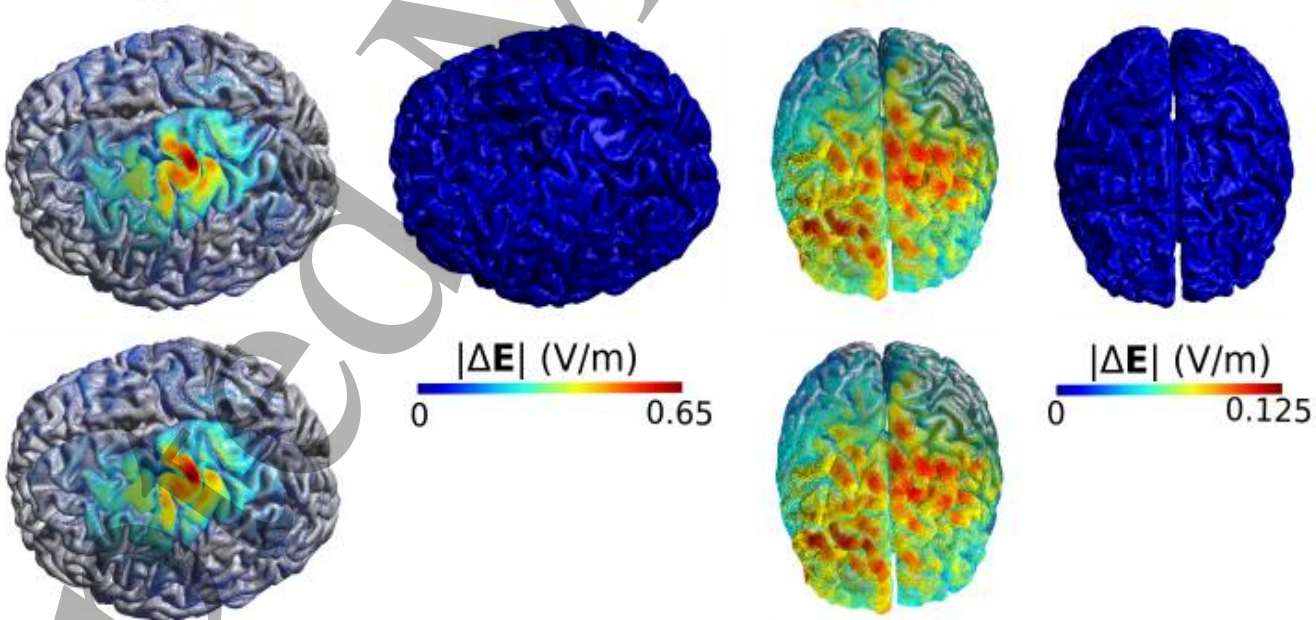

Figure 6: Electric field $\boldsymbol{E}$ and the error relative to the highest resolution model $|\Delta \boldsymbol{E}|$ in the middle cortical layer for the TMS and TES simulations. 
(a)

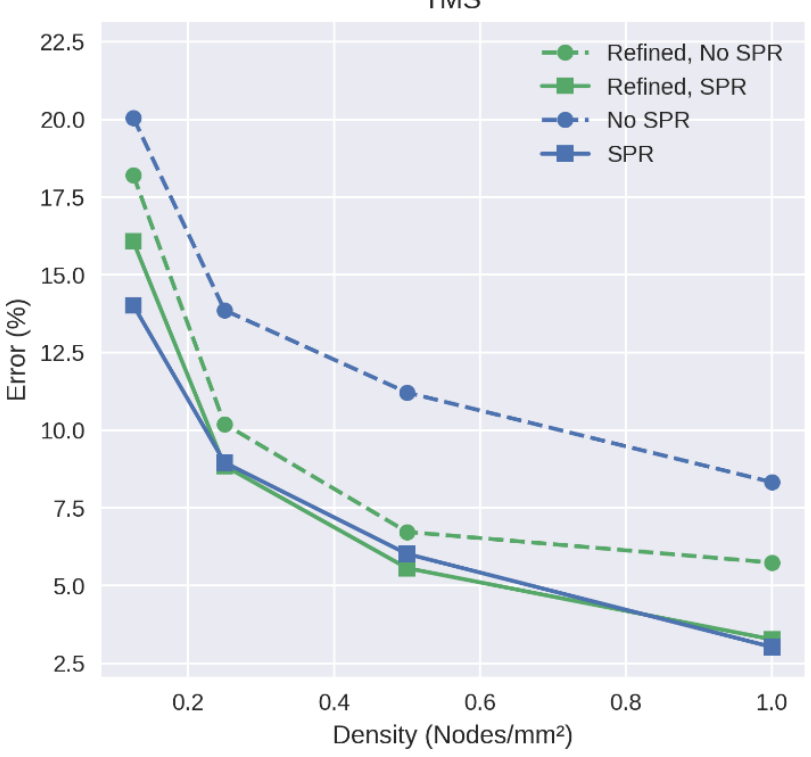

(b)

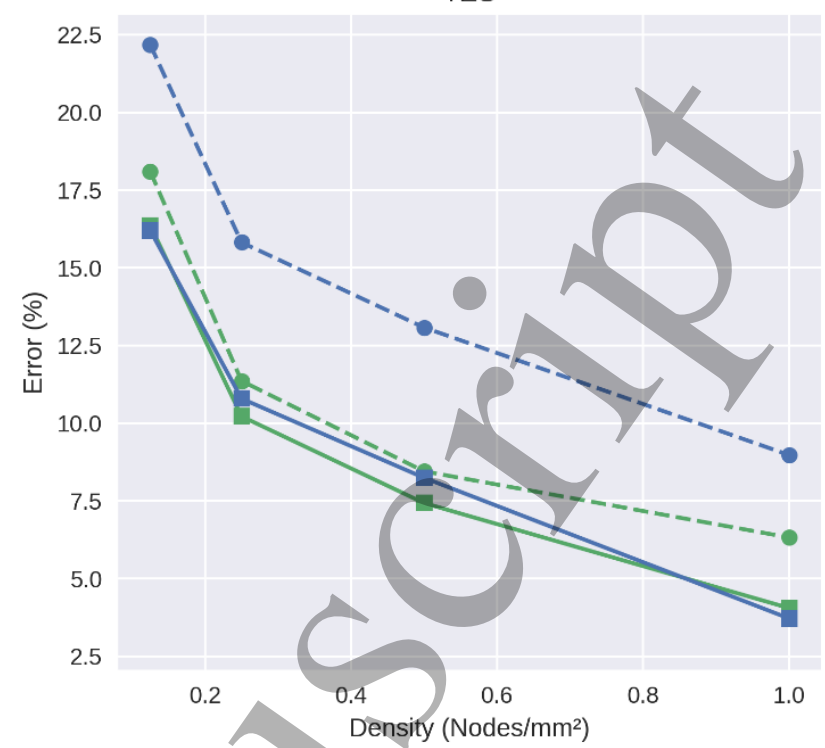

Figure 7: Convergence of the electric field in the middle cortical layer for (a) TMS and (b) TES simulations. Errors were assessed using Equation 6, with the electric field obtained with 2 nodes $/ \mathrm{mm}^{2}$ model as a reference.

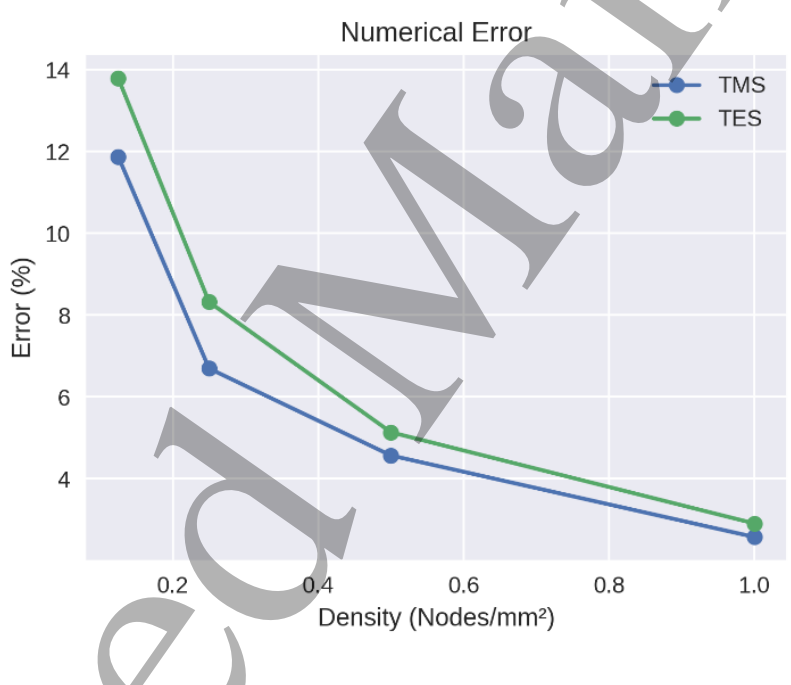

Figure 8: Numerical error estimates obtained using the original and refined versions of the same mesh. SPR was employed during the interpolation of the electric fields 


\subsubsection{Comparison with SimNIBS 2.1}

Figure 9 shows the time used in running an entire simulation (denoted as "All" in the figure legend), including calculating TMS coil $\boldsymbol{A}$ fields and placing TES electrodes, for assembling and solving the FEM systems (denoted as "FEM" in the figure legend) in SimNIBS 2.1 and SimNIBS 3.0, and for running an additional simulation re-using the stiffness matrix and preconditioner (denoted as "Additional FEM" in the figure legend) in SimNIBS 3.0. The calculations were performed on a laptop computer with an Intel i7-7500U processor ( 2 cores, 4 threads), 16 GB of memory, a SSD as harddrive and running Ubuntu Linux 18.04. Times are given as the minimum of ten runs. Given the limited memory in the computer, we were not able to run simulations with the mesh density of 2.0 Nodes $/ \mathrm{mm}^{2}$ using SimNIBS 2.1. We observed markedly lower times in SimNIBS 3.0, together with an apparent change in the asymptotic behavior of the simulation times, with SimNIBS 3.0 performing better on finer meshes. The differences are largely due to the preconditioner used (SimNIBS 2.1 uses an incomplete Cholesky preconditioner and SimNIBS 3.0 uses an AMG preconditioner).

For a mesh of 0.5 Nodes $/ \mathrm{mm}^{2}$, the new FEM approach is about six times faster for assembling and solving a TMS FEM system ( 189.3 versus 29.8 seconds) and almost three times faster in assembling and solving a TES FEM system ( 84.3 versus 29.2 seconds). If we consider the whole process of setting up a simulation, such as interpolating the $\partial \boldsymbol{A} / \partial t$ field in TMS [44], meshing electrodes in TES, and calculating the gradient of the electrical potentials, the speed up is of around five times for a single TMS simulation (196.2 versus 34.8) and around two times for a single TES simulation (140.0 versus 66.3 seconds). Notice that other parts of the code beside the FEM, such as electrode placement in TES, have also been optimized. In scenarios where the head mesh, tissue conductivities and the Dirichlet boundary conditions are kept constant, such as when running simulations with changing coil positions or construction of leadfield matrices for TES optimization [14], the new implementation re-utilizes the stiffness matrices and preconditioners, leading to further speed up. We observed that consecutive simulations take 11.3 seconds for the TMS system and 10.2 seconds for the TES system. This means that, for this head mesh, SimNIBS 3.0 can perform around 14 TMS or five TES field simulations in the same time that SimNIBS 2.1 uses to calculate a single simulation.

Comparing the electric field in the GM volume obtained with SimNIBS 2.1 and SimNIBS 3.0 using Equation 6 , we saw relative differences of $0.052 \%$ and $0.046 \%$ in the mesh with a node density of 0.5 Nodes $/ \mathrm{mm}^{2}$ for TMS and TES, respectively.

Figure 10 shows the total memory requirements for running TMS and TES simulations. SimNIBS 3.0 requires about half the memory of SimNIBS 2.1. We notice that only physical memory was used, even though the memory consumption by the TMS simulation in the mesh of 1.0 nodes $/ \mathrm{mm}^{2}$ density with SimNIBS 2.1 was clóse to the maximum capacity of the system. Please notice that the difference in peak memory usage cannot be attributed to the choice of numerical solver or preconditioner, it is rather dominated by other differences in the implementation details of SimNIBS 3.0, SimNIBS 2.1 and GetDP, in particular related to the assembly of the stiffness matrix. 
Time to Run Simulations

(a)

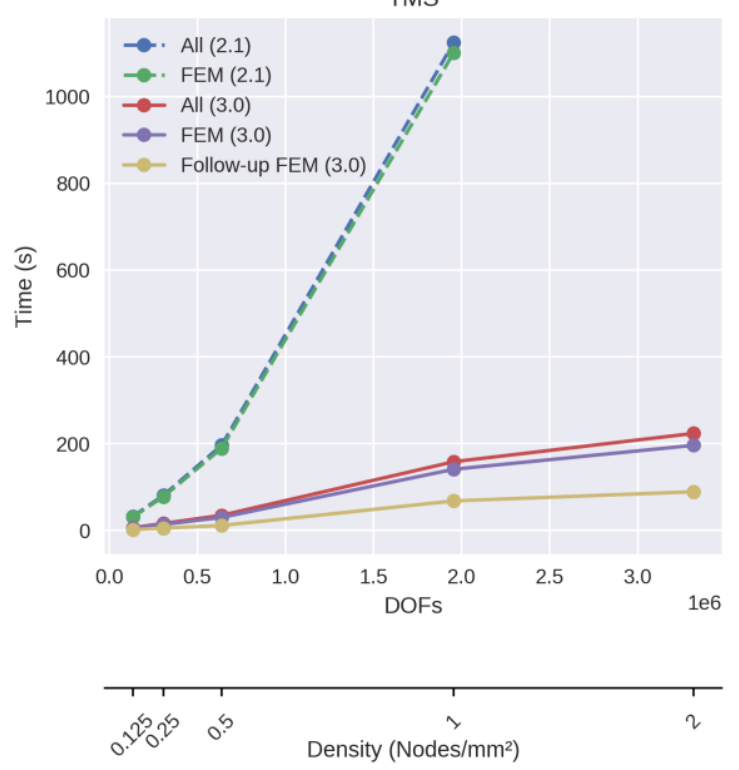

(b)

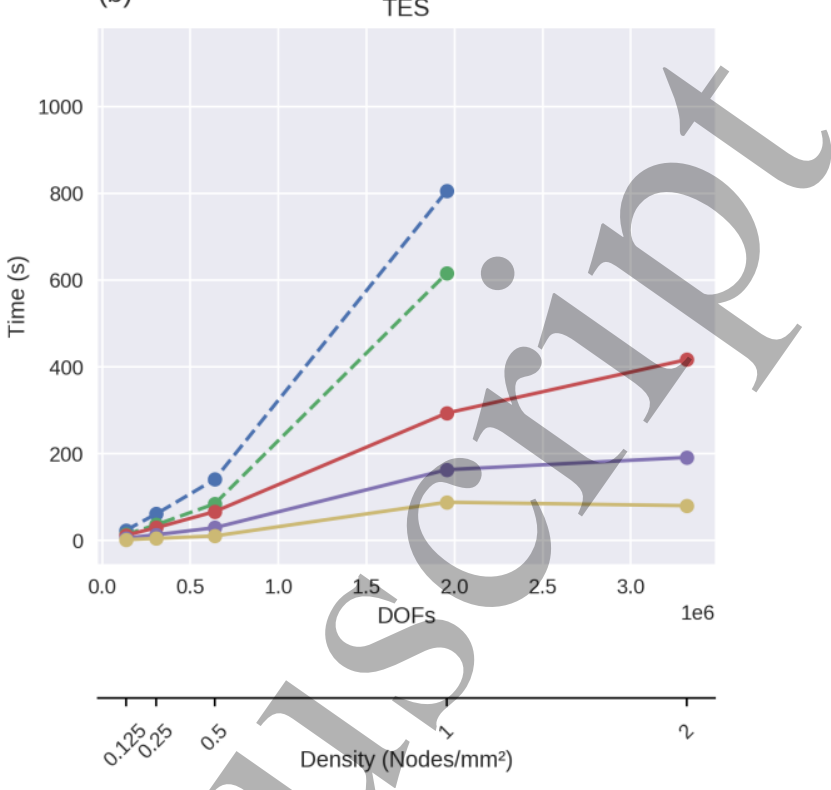

Figure 9: Time to run a (a) TMS and (b) TES simulation in SimNIBS 2.1 and 3.0. In the plots, "All" refers to the whole process of setting up a simulation, assembling and solving the FEM system, "FEM" refers to the process of assembling and solving the FEM system and "Follow-Up FEM" to running a second simulation re-using the matrix and pre-conditioners.

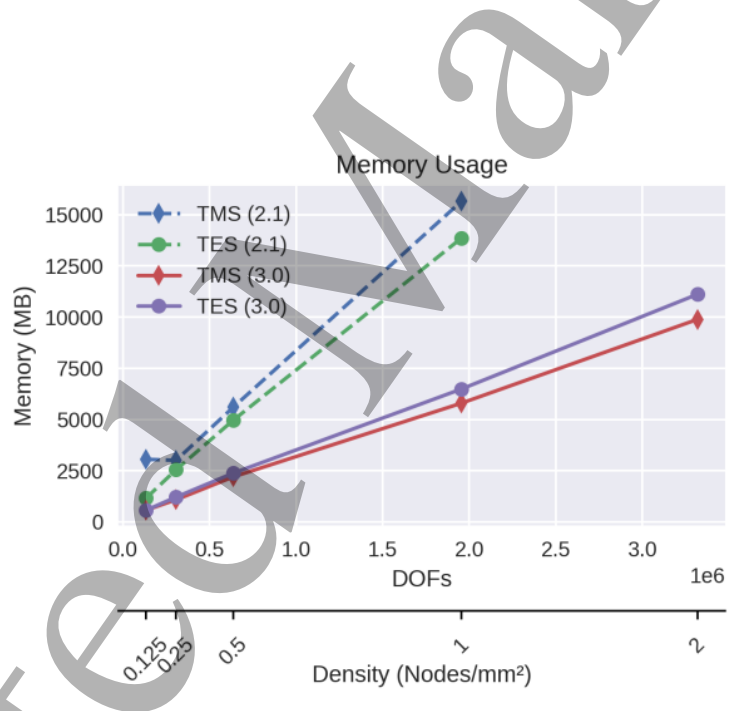

Figure 10: Total memory used by SimNIBS 2.1 and 3.0 for running simulations at various mesh densities

\subsection{Sulcus Phantom}

The electric fields and the errors for the sulcal phantom are shown in Figure 11. Interestingly, the electric field distribution in the sulcus is highly dependent on the set-up, especially for TES. This is due to the large conductivity difference between CSF and GM (1.654 versus $0.275 \mathrm{~S} / \mathrm{m}$ ), which favors current flow through CSF. Thus, when the potentials are set along the top and bottom of the model, the current tend to flow to the bottom of the sulci, where they enter GM. On the other hand, when the potentials are set on the sides of the model, the currents will tend to flow along the CSF layer on the top of the phantom. Generally, the errors tend to be concentrated in the regions of highest 
curvature at the top or at the bottom of the gyrus, and there is a large difference in the magnitude of the errors across the different stimulation set-ups.

Figure 12 shows the average numerical error in the region of interest, calculated using Equation 6 and in relation to the highest resolution model. As in the previous cases, the errors steadily decrease as the model in refined, indicating convergence. As expected from Figure 11, the errors for the TES simulations with the potentials set at the horizontal boundaries are much larger than for the other three set-ups. This is because the electric currents tend to flow along the conductive CSF layer, entering the grey matter at the bottom of the sulcus, where it spreads out again. This effect causes the electric field to be highest in the gray matter of the sulcus fundus and to vary strongly within this relatively small region, so that a higher mesh resolution is required to accurately capture this effect. After doubling the size of the WM layer, the same pattern persisted, showing that this is not an artifact of the boundary condition set-up. It is also interesting to notice that there are no gains obtained by the SPR interpolation of the TMS electric fields in the coarser meshes. This is because the TMS field is focused in the upper part of the gyrus, which in the coarser meshes is composed by a single tetrahedral layer. As there are no internal nodes in this region, our recovery algorithm performs no better than the original result.

In Figure B3 in the Supplementary Material B, we show the errors caused by under-representing sulcus depth, which is a typical artifact in realistic head meshes that occurs for low mesh densities, low-quality input images or when using non-optimal segmentation methods. As expected from the results obtained with the head mesh, the error caused by anatomical misrepresentation ( $25 \%$ to $5 \%$ in the studied range) is much larger than the errors caused by numerical factors ( $11 \%$ to $1 \%$ ). Anatomical misrepresentations are thus likely to contribute substantially to the total error in realistic scenarios. 

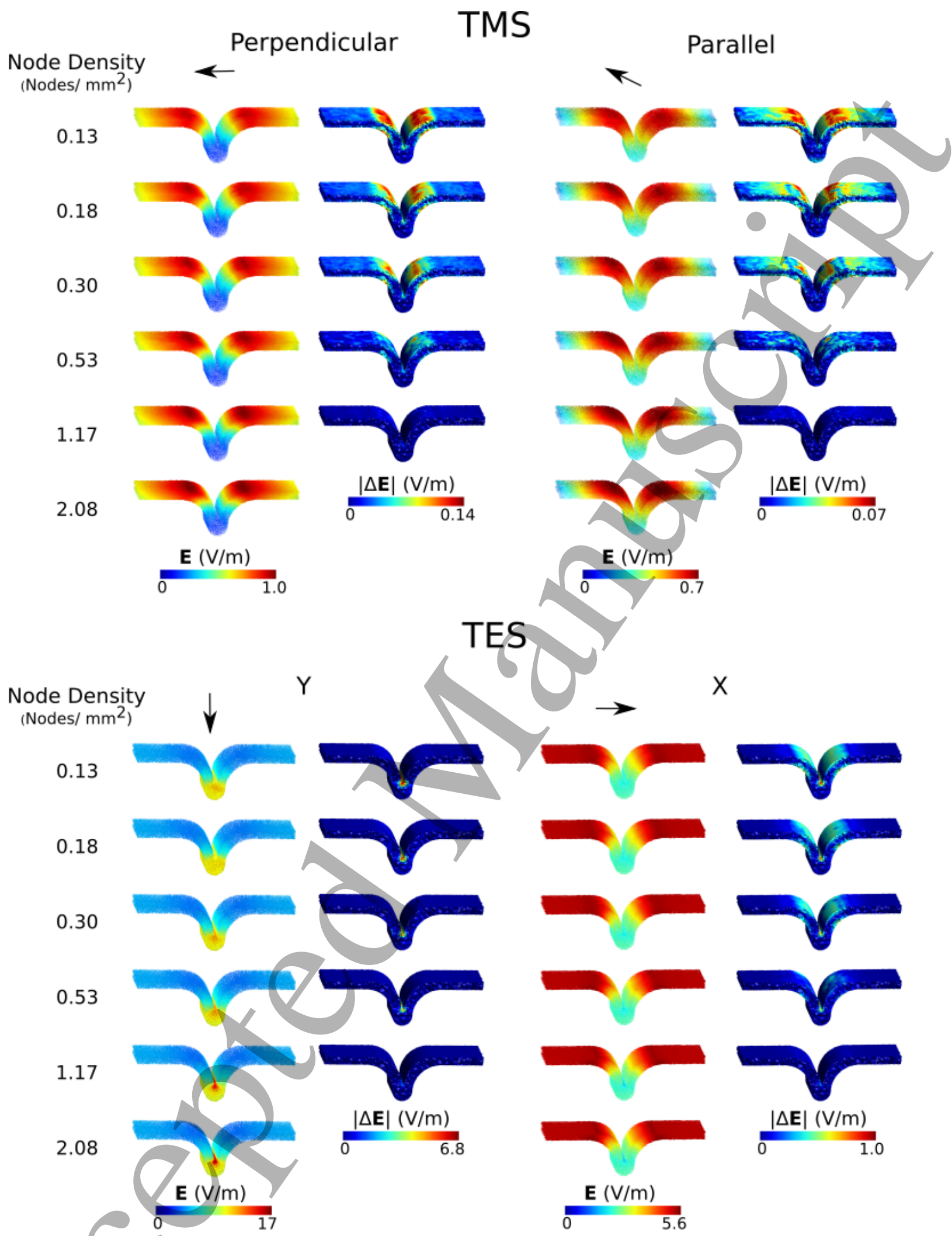

Figure 11: Electric fields and errors in the region of interest of the sulcus phantom. The arrows indicate the main direction of the electric field vectors, and the errors were calculated in relation to the highest resolution model. 
(a)

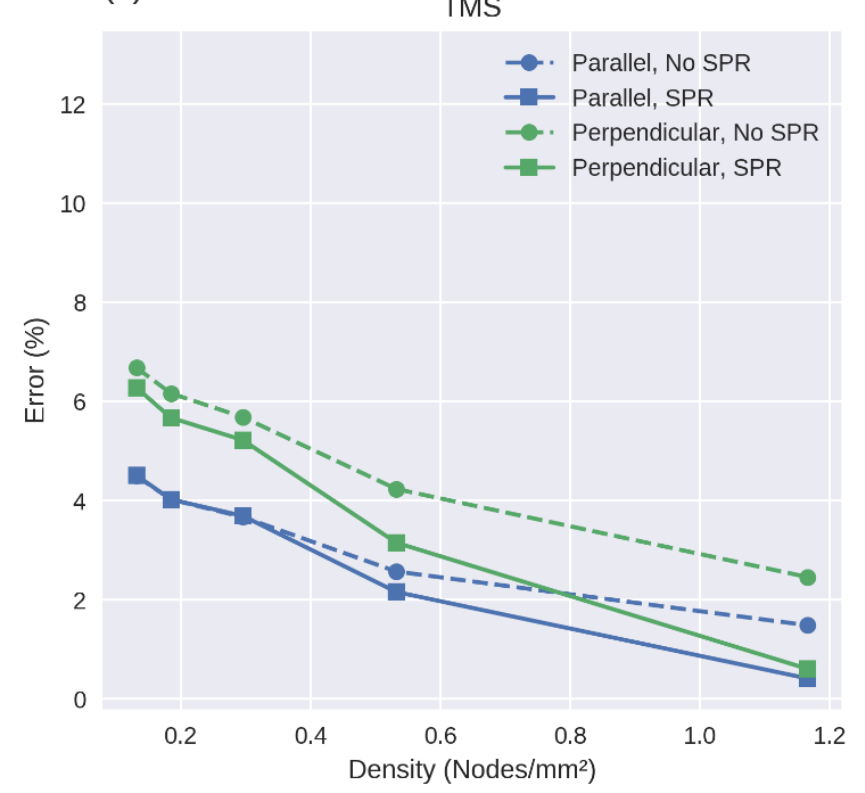

(b)

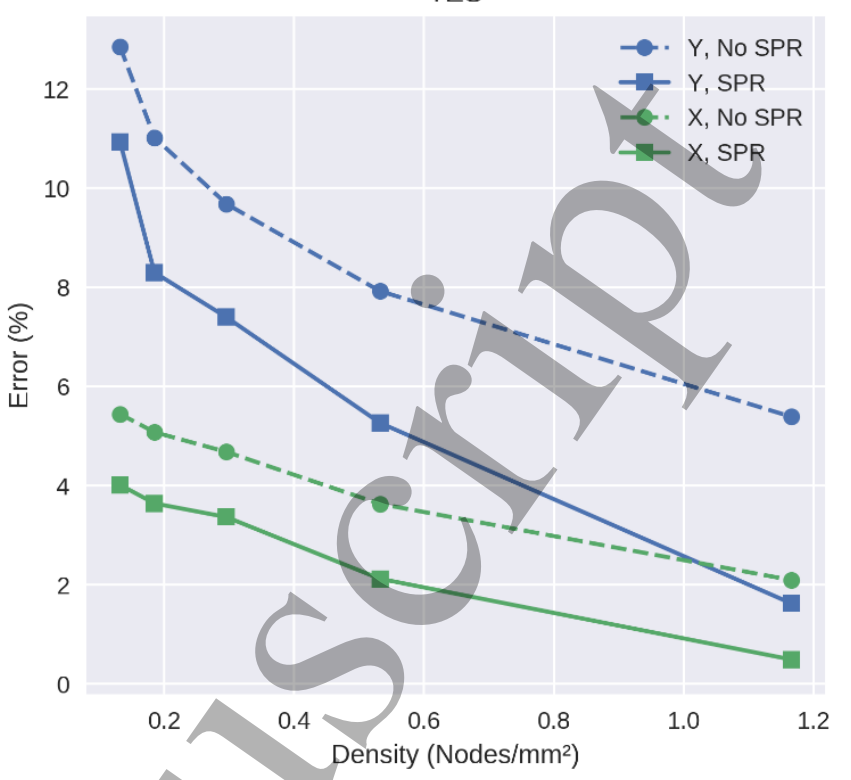

Figure 12: Errors in (a) TMS and (B) TES simulations in the sulcus model, with and without SPR recovery.

\section{Discussion and Conclusion}

In the previous sections we presented an efficient implementation for performing electrical field simulations based on FEM for both TMS and TES. This implementation is available as a part of SimNIBS version 3.0. Validation of the new implementation show that electric fields obtained agree well with analytical solutions of the field in spherical conductors and are very similar to electric fields obtained with a previous implementation (SimNIBS 2.1). However, the new implementation clearly outperforms the previous implementation, runs simulations up to nine times faster and requires up to four times less memory.

Our validations show that TMS and TES simulations converge with increasing anatomical fidelity of the GM layer. This suggests that a detailed representation of the cortical folding is important to obtain accurate results. Furthermore, our results indicate that estimating the elementwise-constant electric field obtained directly from first-order FEM may require a very high node density in order to ensure adequate accuracy, but that this can be somewhat compensated by the use of SPR interpolation. It is also interesting to notice that, while refining the head mesh by splitting the tetrahedra does increase the numerical accuracy of the solution, this does not necessarily improve the overall accuracy when compared to a reference model that is also anatomically more accurate. This shows that increasing the overall model accuracy requires improving both the anatomical fidelity and numerical accuracy.

The data suggests that the choice of an appropriate value for the mesh density is highly dependent on the application. Values as low as 0.25 nodes $/ \mathrm{mm}^{2}$ might be appropriate for qualitative analysis of the electric field distributions. A density of 0.5 nodes $/ \mathrm{mm}^{2}$ is currently set as standard in SimNIBS and seems to give an acceptable trade-off between efficiency and accuracy, with overall errors in the range of $6 \%$ to $8 \%$ and numerical errors below $5 \%$. Values of 1.0 node $/ \mathrm{mm}^{2}$ or higher are needed to ensure overall errors being consistently lower than $5 \%$, as e.g. required for careful analyses of the 
field in the sulci, which are not properly captured in low resolution models. The numerical error is $<3 \%$ in this case. The increased computational efficiency of the new FEM implementation ensures that simulations with these high node densities are no longer prohibited by the increased computational efforts, and can now easily be used on a regular basis. Furthermore, we also show that obtaining good quality MR image is also an important factor for accurately calculating the electric field. As the errors are consistent across stimulation modalities, we do not expect them to exhibit very large variations across simulation set-ups, or between head models of healthy individuals, given that the segmentation is accurate.

We believe that overall simulation errors in the observed range are currently still acceptable, given that other error sources such as segmentation errors [38,45], the uncertainty of the values of the ohmic tissue conductivities [46], putative inaccurate or simplified modeling of the of TMS coil or TES electrode properties and positions [7] can cause errors in or above this range. For example, in a recent study [46], we found standard deviations in maximum electric field values due to uncertainty in tissue conductivities to be around $5 \%$ of the mean value in TMS, but around $20 \%$ of the mean value in TES, and in another study [45], we found the TES electric fields obtained from different head segmentation tools to vary up to $40 \%$. This suggest that future efforts to increase simulation accuracy will have to commonly account for all these factors, rather than focusing on improving the numerical accuracy alone. In addition, considering the state of knowledge on how the electric field modulates neural activity, simulation errors in the reported range do currently not affect the conclusions that can be drawn from the simulation results.

In a related manner, this study is limited to FEM with first order tetrahedral elements. Employing numerical methods other than first order FEM and SPR, such as higher order FEM [21], DG-FEM $[36,47], B E M[48,49]$, and BEM-FMM $[20,21,50]$ will likely lead to better numerical accuracy. However, for all these methods, the results can still only be accurate as far as the head model is accurate, meaning that we still expect the errors, when comparing to a more accurate head model, to quickly reach a lower bound. In line with our ressults, Engwer et. al. [36] noted in the context of DG-FEM for the EEG forward problem that the errors in the solutions seems to be dominated by errors in adequately representing the anatomy, rather than by the solution method (DG-FEM vs. CGFEM).

The timing data shown here was limited to SimNIBS 3.0 and SimNIBS 2.1. However, the BEM-FMM implementation by Makarov et al. [50], shown to outperform SimNIBS 2.1 [20], was recently reported to run in around 90 seconds on a head mesh of 0.6 nodes $/ \mathrm{mm}^{2}$ [51], while we found SimNIBS 3.0 to run in under 40 seconds on a mesh of 0.5 nodes $/ \mathrm{mm}^{2}$ on a computer less powerful than the one used by Makarov et al. Therefore, SimNIBS 3.0 is apparently on par with other state-of -the-art methods for TMS calculations in terms of computational efficiency. However, each method has its advantage, as BEM-FMM offers better numerical accuracy than first order FEM [20], while FEM can handle anisotropic conductivities [52].

In combination, the results for the full head model and the sulcus model indicate that the numerical accuracy of the solutions benefits from an accurate anatomical representation of the tissue boundaries. In particular, the sulcus model shows that a higher mesh density around strongly curved parts of the GM/CSF boundary, where the electric potential can show relative abrupt changes, is helpful in order to avoid local simulation errors around those parts and that the under- 
representation of sulci can cause large errors in the electric field. One course of action suggested by our results would be to improve the surface meshing such that it can preserve the sulci better and increase the node density in regions of high curvature. This could be done by changing the resampling algorithm such that it takes into account local curvature or by other methods such as harmonic maps [53], or isoperimetric higher order elements. Those methods would hopefully allow us to obtain accurate representations of the tissue surface while keeping a small number of elements, and thus improve simulation accuracy at a low computational cost.

In summary, we present a new FEM implementation, which will be made available in SimNIBS 3.0. This implementation leads to up to twenty times faster simulations than in SimNIBS 2.1. This will provide a massive speed up in applications requiring repeated simulations such as calculating several different TMS positions, obtaining leadfields for TES optimization, or performing uncertainty quantification. We also estimated the accuracy for the electric field simulations, demonstrating that that anatomical fidelity is of key importance to obtain accurate results.

\section{Acknowledgements}

This work was suppoted by Lundbeckfonden (grant Nr. R118-A11308), and NovoNordisk fonden (grant Nr. NNF14OC0011413). 
[13] Jung Y J, Kim J H and Im C H 2013 COMETS: A MATLAB toolbox for simulating local electric fields generated by transcranial direct current stimulation (tDCS) Biomed. Eng. Lett. 3 39-46

[14] Dmochowski J P, Datta A, Bikson M, Su Y and Parra L C 2011 Optimized multi-electrode stimulation increases focality and intensity at target. J. Neural Eng.

[15] Guler S, Dannhauer M, Erem B, Macleod R, Tucker D, Turovets S, Luu P, Erdogmus D and Brooks D H 2016 Optimization of focality and direction in dense electrode array transcranial direct current stimulation (tDCS) $\mathrm{J}$. Neural Eng. 13 1-14

[16] Ruffini G, Fox M D, Ripolles O, Miranda P C and Pascual-Leone A 2014 Optimization of multifocal transcranial current stimulation for weighted cortical pattern targeting from realistic modeling of electric fields Neuroimage

[17] Weise K, Di Rienzo L, Brauer H, Haueisen J and Toepfer H 2015 Uncertainty Analysis in Transcranial Magnetic Stimulation Using Nonintrusive Polynomial Chaos Expansion IEEE Trans. Magn. 51

[18] Gomez L J, Yucel A C, Hernandez-Garcia L, Taylor S F and Michielssen E 2015 Uncertainty quantification in transcranial magnetic stimulation via high-dimensional model representation IEEE Trans. Biomed. Eng. 62 361-72

[19] Schmidt C, Wagner S, Burger M, Rienen U Van and Wolters C H 2015 Impact of uncertain head tissue conductivity 
in the optimization of transcranial direct current stimulation for an auditory target J. Neural Eng. 12

[36] Engwer C, Vorwerk J, Ludewig J and Wolters C H 2017 A Discontinuous Galerkin Method to Solve the EEG Forward Problem Using the Subtraction Approach SIAM J. Sci. Comput. 39 B138-64

[37] Rush S and Driscoll D a 1969 EEG electrode sensitivity--an application of reciprocity. IEEE Trans. Biomed. Eng. 16 $15-22$

[38] Nielsen J D, Madsen K H, Puonti O, Siebner H R, Bauer C, Madsen C G, Saturnino G B and Thielscher A 2018 Automatic skull segmentation from MR images for realistic volume conductor models of the head: Assessment of the state-of-the-art Neuroimage c 1-12

Gomez L, Dannhauer M, Koponen L and Peterchev A V 2018 Conditions for numerically accurate TMS electric field simulation bioRxiv 505412

( Dannhauer M, Warfield S K, MacLeod R and Brooks D H 2016 Evaluation of numerical techniques for Imaging (ISBI) vol 2016-June (IEEE) pp 876-80 induced currents in tissues IEEE Trans. Magn. 30 5015-23 J. 63 129-38

Plonsey R and Heppner D B 1967 Considerations of quasi-stationarity in electrophysiological systems Bull. Math. Biophys. 29 657-64 elements: An optimized processing pipeline for the generation and usage of accurate individual head models Hum. Brain Mapp. 34 923-35 stimulation in humans and nonhuman primates Sci. Rep. 6 1-11 Jackson J D 1999 Classical Electrodynamics (John Wiley \& Sons) Zienkiewicz, O.C. , Taylor, R.L, Zhu J . 2013 The Finite Element Method: its Basis and Fundamentals (Elsevier) Zienkiewicz O C and Zhu J Z 1992 The superconvergent patch recovery (SPR) and adaptive finite element refinement Comput. Methods Appl. Mech. Eng. 101 207-24 Knepley M, May D, McInnes L C, Mills R T, Munson T, Rupp K, Sanan P, Smith B, Zampini S, Zhang H and Zhang H 2018 PETSc Web page

Wolters C H, Grasedyck L and Hackbusch W 2004 Efficient computation of lead field bases and influence matrix for the FEM-based EEG and MEG inverse problem Inverse Probl. 20 1099-116 in EIT using algebraic multigrid IEEE Trans. Med. Imaging 24 577-83

Sarvas J 1987 Basic mathematical and electromagnetic concepts of the biomagnetic inverse problem Phys. Med. Biol. 32 11-22 mimicking transcranial magnetic stimulation (TMS) J. Neural Eng. 1-3

[40] Taubin G 1995 Curve and surface smoothing without shrinkage Proceedings of IEEE International Conference on 
Computer Vision (IEEE Comput. Soc. Press) pp 852-7

[41] Dahnke R, Yotter R A and Gaser C 2013 Cortical thickness and central surface estimation Neuroimage 65 336-48

[42] Dubuisson M-P and Jain A K 2002 A modified Hausdorff distance for object matching Proceedings of 12th International Conference on Pattern Recognition vol 1 (IEEE Comput. Soc. Press) pp 566-8

[43] Geuzaine C 2007 GetDP: a general finite-element solver for the de Rham complex PAMM 7 1010603-4

[44] Madsen K H, Ewald L, Siebner H R and Thielscher A 2015 Transcranial magnetic stimulation: An automated procedure to obtain coil-specific models for field calculations Brain Stimul. 1-4

[45] Puonti O, Saturnino G B, Madsen K H and Thielscher A 2019 Comparing and Validating Automated Tools for Individualized Electric Field Simulations in the Human Head bioRxiv 611962

[46] Saturnino G B, Thielscher A, Madsen K H, Knösche T R and Weise K 2018 A principled approach to conductivity uncertainty analysis in electric field calculations Neuroimage

[47] Piastra M C, Nüßing A, Vorwerk J, Bornfleth H, Oostenveld R, Engwer C and Wolters C H 2018 The discontinuous Galerkin finite element method for solving the MEG and the combined MEG/EEG forward problem Front. Neurosci. 12

[48] Nummenmaa A, Stenroos M, Ilmoniemi R J, Okada Y C, Hämäläinen M S and Raij T 2013 Comparison of spherical and realistically shaped boundary element head models for transcranial magnetic stimulation navigation Clin. Neurophysiol. 124 1995-2007

[49] Salinas F S, Lancaster J L and Fox P T 2009 3D modeling of the total electric field induced by transcranial magnetic stimulation using the boundary element method Phys. Med. Biol. 54 3631-47

[50] Makarov S, Noetscher G, Raij T and Nummenmaa A 2018 A Quasi-Static Boundary Element Approach with Fast Multipole Acceleration for High-Resolution Bioelectromagnetic Models IEEE Trans. Biomed. Eng. 02114

[51] Makarov S N, Noetscher G M, Burnham E H, Pham D N, Htet A T, de Lara L N, Raij T and Nummenmaa A 2019 Software Toolkit for Fast High-Resolution TMS Modeling bioRxiv

[52] Wolters C H, Anwander a., Tricoche X, Weinstein D, Koch M a. and MacLeod R S 2006 Influence of tissue conductivity anisotropy on EEG/MEG field and return current computation in a realistic head model: A simulation and visualization study using high-resolution finite element modeling Neuroimage 30 813-26

[53] Remacle J-F, Geuzaine C, Compère $\mathrm{G}$ and Marchandise E 2010 High-quality surface remeshing using harmonic maps Int. J. Numer. Methods Eng. n/a-n/a 